\title{
Modeling of Mitral Chordae's Length In Echocardiography As A Function of Their Manual Measurement In The Operating Room
}

\section{Mesut GUN ( $\square$ gun.mesut@chu-amiens.fr )}

CHU Amiens-Picardie Pôle Coeur Thorax Vaisseaux: Centre Hospitalier Universitaire Amiens-Picardie Pole Coeur Thorax Vaisseaux https://orcid.org/0000-0003-1705-1122

\section{Misbaou BARRY}

CHU Amiens-Picardie Pôle Coeur Thorax Vaisseaux: Centre Hospitalier Universitaire Amiens-Picardie Pole Coeur Thorax Vaisseaux

\section{Yohann BOHBOT}

CHU Amiens-Picardie Pôle Coeur Thorax Vaisseaux: Centre Hospitalier Universitaire Amiens-Picardie Pole Coeur Thorax Vaisseaux

\section{Christophe TRIBOUILLOY}

CHU Amiens-Picardie Pôle Coeur Thorax Vaisseaux: Centre Hospitalier Universitaire Amiens-Picardie Pole Coeur Thorax Vaisseaux

\section{Gilles TOUATI}

CHU Amiens-Picardie Pôle Coeur Thorax Vaisseaux: Centre Hospitalier Universitaire Amiens-Picardie Pole Coeur Thorax Vaisseaux

\section{Research article}

Keywords: Echocardiography Measurement, Manual Measurement, Correlation, linear analysis, prediction, mitral tendinous chordae, mitral valve repair

Posted Date: July 20th, 2021

DOl: https://doi.org/10.21203/rs.3.rs-673964/v1

License: (c) (1) This work is licensed under a Creative Commons Attribution 4.0 International License. Read Full License

Version of Record: A version of this preprint was published at Journal of Cardiothoracic Surgery on April 4th, 2022. See the published version at https://doi.org/10.1186/s13019-022-01816-8. 


\section{Abstract}

\section{Objective}

In mitral insufficiency, trans-esophageal echocardiography (TEE) analysis of the mitral valve is an indispensable and irreplaceable examination to establish precisely the type of surgical repair to be performed and the exact length of neo-chordae to be used for an anatomical repair. The aim of our study is to find a predictive model of the Echographic Measurement (EM) variable according to the Manual Measurement (MM) variable of the mitral valve chordae, when the echocardiography measurement is not feasible.

\section{Patients and methods}

This is a retrospective study on 191 patients undergoing mitral valve repair. The sex ratio (M/F) is 2.13 (130 men and 61 women). The collection of data of mitral chordae measurements performed echographically in preoperatively conditions, and then manually in intraoperatively conditions from January 2008 to December 2016 was made from the medical records of patients at the cardiology and cardiac surgery department of the University Hospital Center of Amiens in Picardy.

\section{Results}

For this study 191 patients of mean age of $68 \pm 13$ years were included. The averages of the MM and EM of the mitral chordae were respectively $23 \pm 2.5 \mathrm{~mm}$ and $24 \pm 2.4 \mathrm{~mm}$. The Pearson correlation coefficient was 0.897 ( $p$-value $<10 \rrbracket^{4}$ ) showing a strong positive correlation between MM and EM. The results of the linear regression allow us to found the following mathematical model : $E M=0.87 x M M+4$.

\section{Conclusions}

When patients have a contraindication to transesophageal echocardiography or when TEE is not feasible, manual measurement is performed during the surgery. By using the values obtained (MM) in the model, it is possible to predict the corresponding echographic measurements. This allows us to achieve the mitral tendinous chordae substitution with a very high precision.

\section{Trial registration}

Retrospectively registered.

\section{Introduction}

The implantation of neo-chordae, consisting of replacing broken or elongated chordae with artificial chordae made of four-needle tresses of Gore-Tex ${ }^{\circledR}$, is a recent technique for repairing the mitral valve [1] allowing, without any valve resection, to effectively correct mitral leakage while preserving the mobility of the two anterior and posterior valve leaflets. The length of these braids necessary to optimally correct 
mitral prolapse can be measured : either manually with the heart stopped during the extra-corporeal circulation (ECC), hypothermic and bloodless, without any pre- and post-load conditions, or by transesophageal echocardiography (TEE) performed in immediate preoperative which allows to predict, in a more physiological way, the length of these neo-chordae [2]. The echocardiographic measurement, made in systole during valve closure, corresponds to the distance between the top of the papillary muscle and the free edge of the non-prolapsed valve, opposite the valve leaflet, where the chordae is broken or elongated. This precise and reliable method of echocardiographic measurement should be preferred because it is carried out under physiological conditions of myocardial contractility. Some patients, however, have a contraindication to TEE :

- esophageal pathologies (stenosis, diverticulum, tumor, varicose veins, scleroderma),

- unstable lesions of the cervical spine,

- history of chest radiotherapy,

- massive hematemesis, progressive upper gastrointestinal haemorrhagic lesion, gastric or esophageal perforation,

- recent esogastric surgery,

- situations leading to the evaluation of a benefit / risk (shock, severe hypoxia),

- unfavorable anatomical conditions (poorly filled cardiac cavities, abnormal rotation of the atrioventricular floor, difficulty in exposing the papillary muscles) [3],

- anatomical variation in the number and shape of the pillars $[7,8]$

All these contraindications require the performance of a manual intraoperative measurement [2]. The objective of this work was to find a model to predict echocardiographic measurement (EM) from manual measurement $(\mathrm{MM})$.

\section{Patients And Methods}

\section{1 Study population}

It is an observational, retrospective and monocentric study aiming to identify a model allowing to predict the EM from the MM in a population of patients having benefited from a surgical mitral valve repair by implantation of neo-chordae at the University Hospital Center of Amiens from Picardy.

The cordage measurement was performed manually and echocardiographically in 191 patients with severe mitral insufficiency and who had undergone mitral reconstruction surgery. 
The study protocol was approved by the ethics committee of the University Hospital Center of Amiens in Picardie in France on 29 January 2013.

\section{1 Study population}

We measured these chordae first by trans-esophageal echocardiography (TEE), under general anesthesia as an immediate preoperative, then by manual intraoperative measurement with a graduated rule (heart stopped by cardioplegia, left atrium open). Myocardial protection was ensured by hyperpotassic and hypothermic cardioplegia $\left(4^{\circ} \mathrm{C}\right)$. The distance measured corresponds to the distance between the top of the papillary muscle and the free edge of the non-prolapsed valve opposite to the prolapse (ex. for segment P2 prolapse, measurement of the distance between the tip of the posteromedial pillar and the free edge of segment $A 2$ ).

All the pre- and intraoperative data (MM, EM, age and sex) from the month of January 2008 to the month of December 2016 were extracted from the medical files of the patients of the medical and surgical cardiology department.

\section{3 Statistical analysis}

We analyzed the data using SAS statistical version 7.1. In the descriptive study, we calculated absolute frequencies and relative frequencies (percentages) for the qualitative variables. We calculated means and standard deviations for the quantitative variables and analyzed the data to study the skewness of the distributions and outliers. We analyzed the normality of the two distributions by the tests of Shapiro-Wilk, Kolmogorov-Smirnov, Cramer-Von Mises and Anderson-Darling as well as their spread around the central value by checking the asymmetry coefficient (Skewness) and the coefficient flattening (Kurtosis) and another graphical appreciation of the fit, the quantile-quantile diagram, for the two distributions: manual measurement (MM) and echocardiographic measurement (EM). In the analytical study, the links between two quantitative variables were studied by the Pearson correlation coefficient. The univariate analysis was performed using simple linear regression to determine the mathematical model of the regression line.

In all the statistical tests, the significance level was fixed at a probability $a: 0.05$.

\section{Results}

\section{1 General characteristics of the population}

In total, we studied 191 patients with a male predominance (68\%). The sex ratio (M / F) was 2.13 (130 men and 61 women). The mean age was $68 \pm 13$ years (21-91 years). Table 1 summarizes the characteristics of the population studied.

Table 1. Characteristics of study population $(\mathrm{N}=191)$ 


\begin{tabular}{|l|l|}
\hline Characteristics & Values \\
\hline Age - mean \pm SD (years) & $68 \pm 13$ \\
Sex-ratio (M/F) & 2.13 \\
MM - mean \pm SD (mm) & $23 \pm 2.50$ \\
EM - mean (mm) & $24 \pm 2.40$ \\
MM - minimum (mm) & 16 \\
MM - maximum (mm) & 31 \\
EM - minimum (mm) & 17 \\
EM - maximum (mm) & 31.5 \\
\hline
\end{tabular}

\section{Abreviations : MM, Manual Measurement ; EM, Echographic Measurement ; mm, milimeter}

The means of preoperative echocardiographic (EM) measurements (Fig. 1) and manual intraoperative (MM) measurements (Fig. 2) of the mitral chordae were $23 \pm 2.5$ millimeters and $24 \pm 2.4$ millimeters respectively.

\section{2 Normality for manual measurement and echocardiographic measurement}

We calculated the parameters (mean, standard deviation) of the variable MM and the variable EM (table 1).

The normality of the quantitative variables MM and EM was verified by the tests of Shapiro-Wilk, Kolmogorov-Smirnov, Cramer-Von Mises and Anderson-Darling. The quantitative results which are detailed in Table 2 revealed perfect normality ( $p$-value $>0.05$ ) for each of the tests which therefore allowed acceptance of the null hypothesis $\mathrm{H} 0$ and rejection of the alternative hypothesis $\mathrm{H} 1$. 


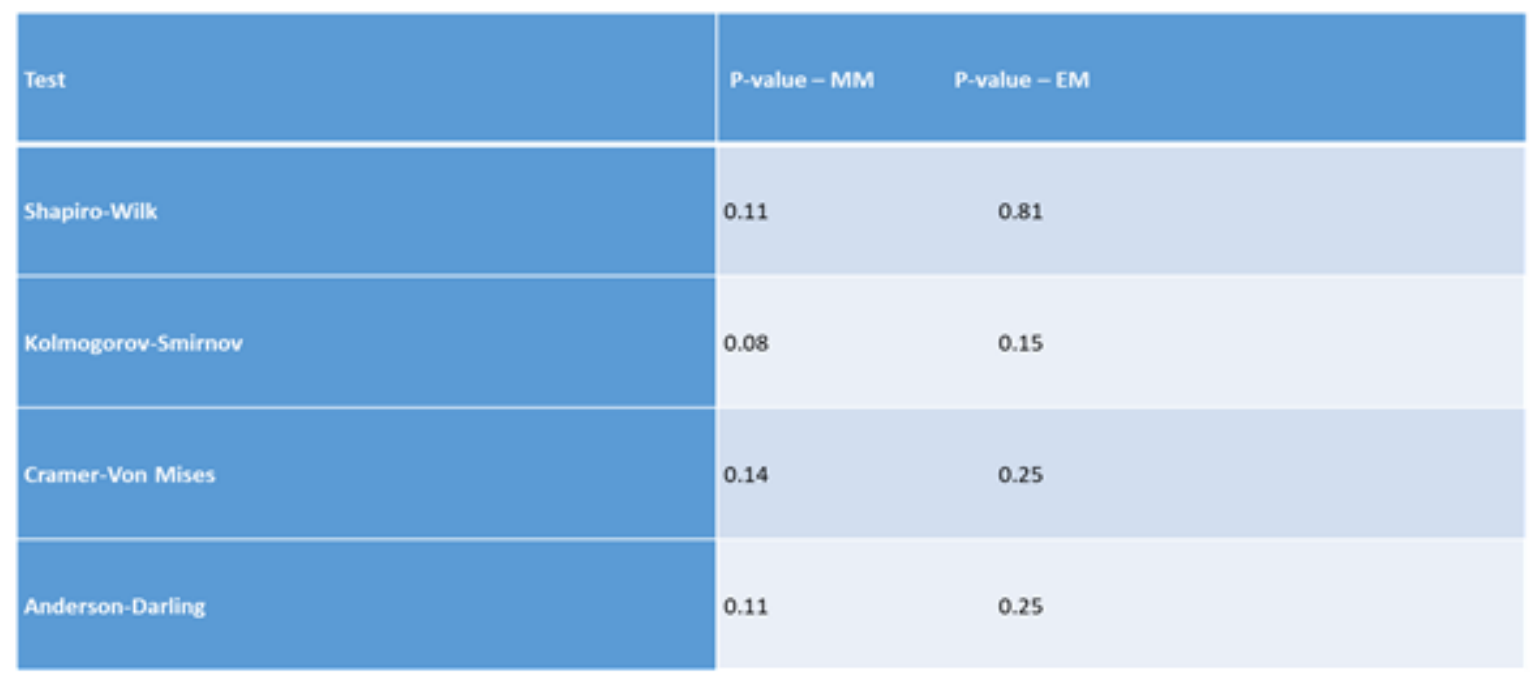

Their spread around the central value was checked by the asymmetry coefficient (Skewness $\rightarrow$ SMM: 0.33; SME: 0.08) and the flattening coefficient (Kurtosis $\rightarrow$ KMM: 0.61; KME: 0, 17) (Fig. 3, Fig. 4).

\section{3 Correlation between echocardiographic measurement and manual measurement}

We then verified and studied the statistical significance by a correlation with the Pearson correlation coefficient ( $r$ ). For this, we posed the hypothesis $\mathrm{HO}$ which signifies an independence (the inexistence of relation, $\mathrm{HO}:|\mathrm{r}|=0$ ) against the alternative hypothesis $\mathrm{H} 1$ signifying a dependence between the two variables echographic measurement and manual measurement of the mitral cords $(\mathrm{H} 1:|r| \neq 0)$, we set a probability of 0.05 as risk of error for the rejection of $\mathrm{H} 0$.

We looked for the absolute value of the Pearson correlation coefficient r (MM, EM) in the corresponding table of the correlation coefficient which corresponds to 0.1946 for $N>100$ and a: 0.05 . (See the table of correlation coefficient in the annex).

We calculated the theoretical value of the correlation coefficient $r$ which is 0.897 . We thus rejected $\mathrm{H} 0$ and retained $\mathrm{H} 1$ because $r$-critical $(0.1946)$ is less than $r$-calculated $\left(0.897\right.$ with $\left.p<10 \mathbb{\bigotimes}^{4}\right)($ Table 3$)$.

\section{Table 3. Pearson Correlation Statistics (r)}

\begin{tabular}{lllllcl}
\hline Variable & With variable & $\mathrm{N}$ & Sampling correlation & Confidence interval (95\%) & p-Value \\
\hline EM & MM & 191 & 0.897 & 0.865 & 0.921 & 0.0000
\end{tabular}

We have shown that the correlation coefficient is statistically significant because the calculated $r$ is much higher than the critical $r$ with a risk a: 0.05 and with degrees of freedom N-2 (191 inclusions-2: 189).

We therefore highlighted a strong positive relationship between echocardiographic measurements and manual measurements of the mitral cord. The coefficient of determination $r^{2}$ is 0.805 (Fig. 5).

\section{4 Simple linear regression}


We therefore proceeded to analyze a simple linear regression in order to model the relationship between our two variables (MM and EM).

The relationship between the two variables is linear (the point cloud is best summarized by a line of equation $Y=a X+b)$. The application condition is verified, it is therefore possible to use the correlation coefficient and the simple linear regression to quantify the link between the 2 variables (EM as dependent variable and MM independent variable).

Graphical analysis shows that the residues visually follow a normal distribution (Fig. 6). Errors are therefore distributed normally. The coefficient of determination $\mathrm{R}^{2}$ which measures the percentage of variability in ultrasound values according to manual values is $80.5 \%$, so the model seems adequate.

The adjustment curve of the dependent variable $(\mathrm{EM})$ compared to the independent variable $(\mathrm{MM})$ clearly shows good positive linearity (Fig. 7).

The results of the linear regression lead us to the following mathematical model:

$\mathrm{Y}=\mathrm{aX}+\mathrm{b} \rightarrow \mathrm{Y}=0.87 \cdot \mathrm{X}+4$

Where $Y$ is the dependent variable: echocardiographic measurement (EM) to predict; $X$ is the independent variable: manual measurement ( $\mathrm{MM})$ as a predictor variable; $a$ is the slope of the regression line and $b$ is the intercept.

This model gave an equation by the regression line with a better fit which is as follows : $E M=0.87 \cdot M M$ +4 .

The EM model $=0.87 \cdot \mathrm{MM}+4$, allows us to predict, for example, that for a manual measurement of a 20 $\mathrm{mm}$ chordae, the estimated echocardiographic measurement would be $21.4 \mathrm{~mm}$, which is a significant correction for good surgical repair of the mitral valve.

\section{Discussion}

The need for a reliable measurement of chordae in ventricular dynamics is necessary for a perfect anatomical repair of the mitral valve.

Esophageal echocardiographic measurement is sometimes contraindicated or impossible to perform.

These contraindications were listed previously in our introduction, however unfavorable anatomical conditions are also known $[7,8]$. If $100 \%$ of the hearts studied in different studies show the consistency of an anterior papillary muscle and a posterior papillary muscle, there is, however, significant variability in the number and shape of the bellies of these papillary muscles.

In order to accurately predict a measurement, it is essential to use an appropriate methodology to perform adequate modeling. 
Several authors, $[2,3,4,5,6]$ presented works with different methods of study and analysis.

In this article, we have suggested criteria for the design of studies aiming to collect data to arrive at a standard model whose application makes it possible to predict the length of the echographic measurements on heart observed in dynamics from the corresponding lengths of our manual measurements on a stopped heart. We also presented an analysis strategy to produce graphs from our data. This modeling of our manual measurement is necessary. It allows us to carry out with very great precision our substitution of chordae allowing not only to effectively correct mitral leakage but also to obtain better valve clearance by preserving a harmonious bivalvular movement [1]. Manual measurement remains an anatomical measurement, however it cannot be used as such during the production of artificial chordae, it must be corrected by the proposed formula: EM : $0.87 \cdot M M+4$, in order to obtain perfect coaptation.

The MM always remain lower than the EM which are carried out under the physiological conditions of myocardial contractility.

\section{Conclusion}

The necessary length of Gore-Tex® braids should be estimated as far as possible using transesophageal echocardiography because of the more physiological nature of the measurement and the risk of residual mitral leakage in the event of a measurement estimated manual. When patients have a formal ETO contraindication, or when ETO echocardiographic measurement is impossible, it is therefore necessary to be able to accurately predict this echocardiographic measurement from manual measurement in order to perform the substitution of chordae with very high precision.

If the TEE is often necessary to predict the feasibility of the mitral plasty, it is no longer essential in immediate preoperative to predict the size of neo-chordae necessary for repair. Indeed, the modeling of the length of the mitral valve cords by the $\mathrm{EM}=0.87 \cdot \mathrm{MM}+4$ model eventually makes it possible to overcome this echocardiographic measurement.

This modeling takes into account the dynamic conditions (filling of the beating heart) which are lacking during Manual Measurements during the operation.

Therefore, in case of contraindication to ETO or in case of difficult measurements in ETO, the application of the model obtained will make it possible to perform a mitral valvuloplasty with reliable results.

\section{Abbreviations}

TEE : Trans-Esophageal Echocardiography ; EM : Echographic Measurement; MM : Manual Measurement ; ECC : Extra-Corporeal Circulation ; SMM : Skewness Manual Measurement ; KMM : Kurtosis Manual Measurement ; Ho : Null Hypothesis ; H1 : Alternative Hypothesis ; r : Correlation Coefficient ; N : Total Population; $a$ : Alpha risk. 


\section{Declarations}

\section{Ethics approval and consent to participate}

The study protocol was approved by the Local Ethic Committee of the University Hospital of Amiens of Picardie, France, for the handling and analysis data (CEERNI-ID number : 114-January 29, 2013).

The trial has been registered under the following clinical trial registry number (clinicaltrials.gov) : NCT03113552 and Protocol ID : PI2013_843_0002 with Primary Completion Date : July 4, 2025.

The declaration number to the Paris-CNIL (8 rue vivienne 75083 Paris, cedex 2) : 1665485.

\section{Consent for publication}

Not applicable.

\section{Availability of data and materials}

The datasets used and analysed during the current study are available from the corresponding author on reasonable request.

\section{Competing interests}

The authors declare that they have no links of interest.

\section{Funding}

None.

\section{Authors' contributions}

Mesut GUN, Eng : ideation, database, writing, statistic; Misbaou BARRY, MD, PhD : supervision, revision, writing, Manual Measurements; Yohann BOHBOT, MD, PhD : supervision, revision, Echographic Measurements; Christophe TRIBOUILLOY, MD, PhD : supervision, revision, Echographic Measurements; Gilles TOUATI, MD, PhD : writing, supervision, revision, Manual Measurements. The authors read and approved the final manuscript.

\section{Acknowledgements}

Not applicable.

\section{Authors' information}

Mesut GUN : Research engineer for the Heart-Chest-Vascular Center, University Hospital Center, Amiens, France; Misbaou BARRY : Heart-Chest-Vascular Surgery Center, University Hospital Center, Amiens, France; Yohann BOHBOT : Head of Echographic Laboratory, University Hospital Center, Amiens, France; 
Christophe TRIBOUILLOY : Head of the Heart-Chest-Vascular Center, University Hospital Center, Amiens,

France; Gilles TOUATI : Heart-Chest-Vascular Surgery Center, University Hospital Center, Amiens, France.

\section{References}

1. Touati G, Barry M, Foulon P, Havet E, Laude M, Fournot L, et al. Biométrie des cordages de la valve mitrale: quand l'anatomie vole au secours de la chirurgie de reconstruction. Article in Morphologie. 2014;98(322):115-11.

2. Barry M, Touati G, Foulon P, Ben Ammar M, Trojette F, Havet E, et al. Appréciation de la longueur des cordages mitraux: comparaison entre mesure échographique et mesure manuelle. Applications en chirurgie cardiaque. Article in Morphologie. 2017;101(335):201.

3. Flachskampf FA, Badano L, Daniel WG, Feneck RO, Fox KF, Fraser G, et al. (2010). Recommendations for trans-oesophageal echocardiography. European Journal of Echocardiography 11, 557 - 556.

4. Altman DG. Pratical statistics for Medical Research. London: Chapman and Hall; 1991. p. 136.

5. Altman DG. Construction of age-related reference centiles using absolute residuals. statistics in Medicine. 1993;12:917-24.

6. Healy MJR et Tanner JM. Standards for growth increments. Am J Hum Biol. 1993;5:147-8.

7. Gumusalan Y, Ozbag D, Ozden H, Saruhan B, Demirant A. The comparative investigation of left ventricle papillary muscle arteries in different species. Saudi Med J. 2006;27(6):826-32.

8. Ozbag D, Gumusalan Y, Demirant A. The comparative investigation of morphology of papillary muscles of left ventricle in different species. Blackwell Publishing Ltd Int $\mathrm{J}$ Clin Pract. 2005;59(5):529-36.

\section{Figures}




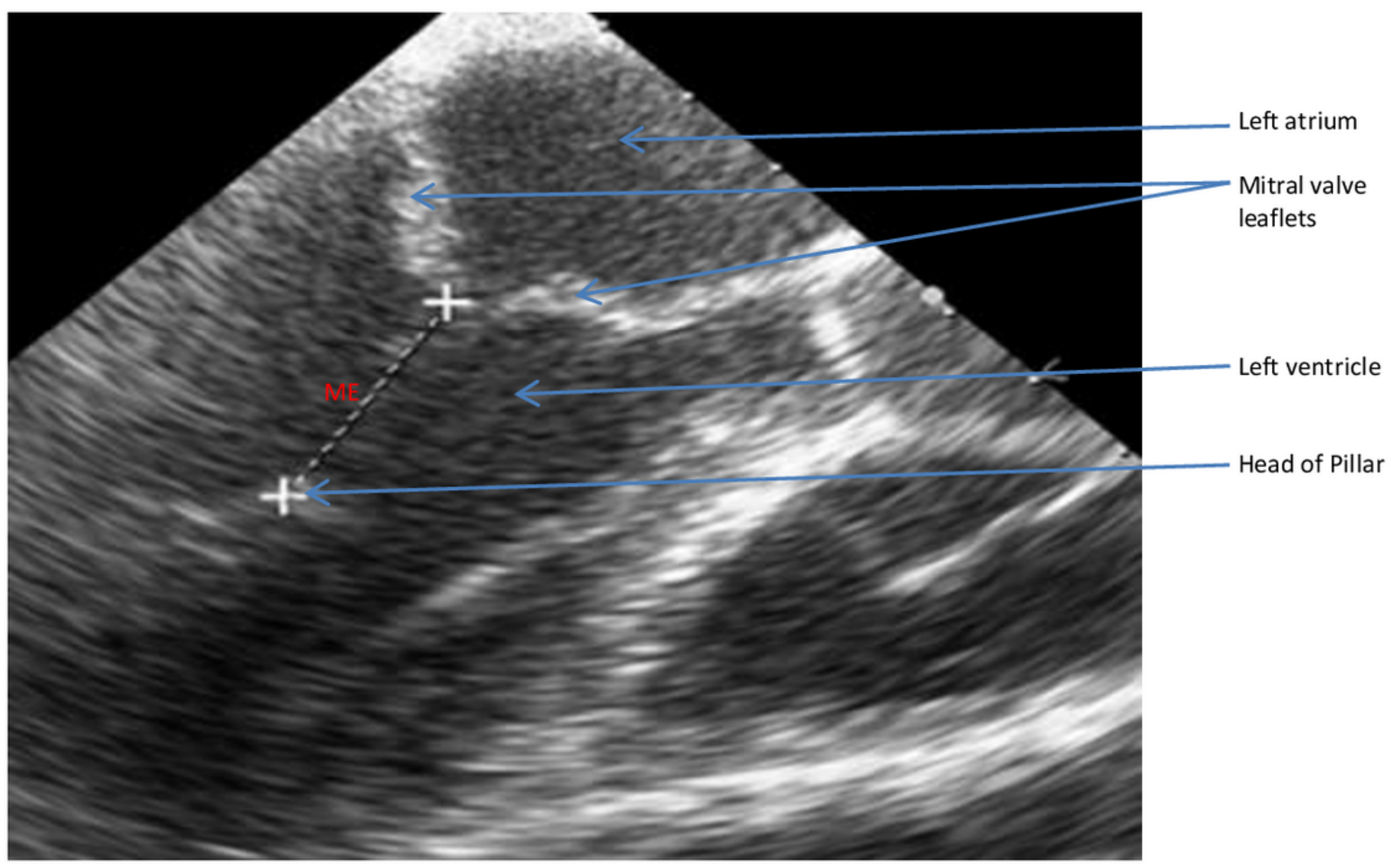

\section{Figure 1}

Echographic Measurement (ME) of the mitral chordae 


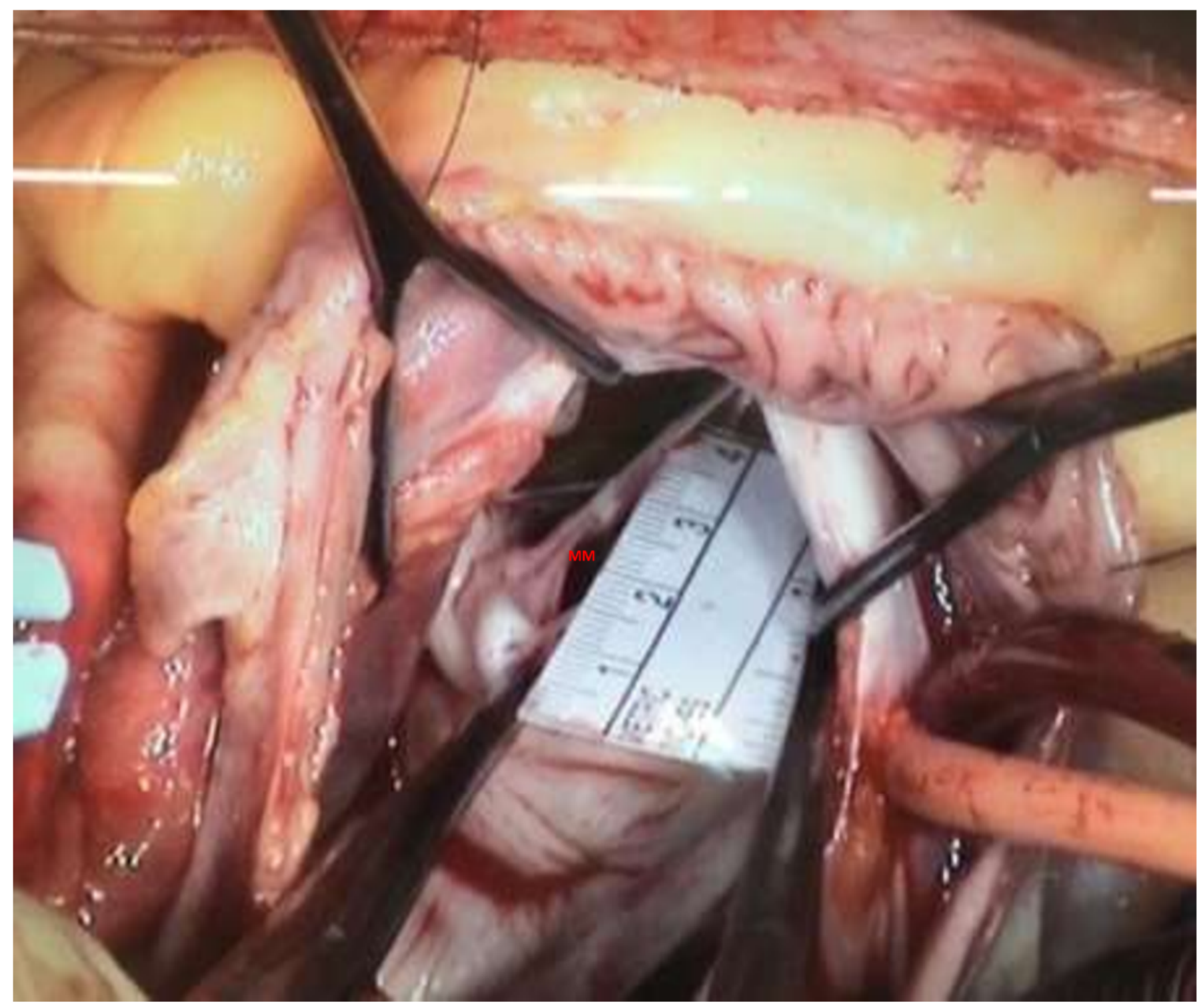

Figure 2

Manual Measurement (MM) of mitral chordae 


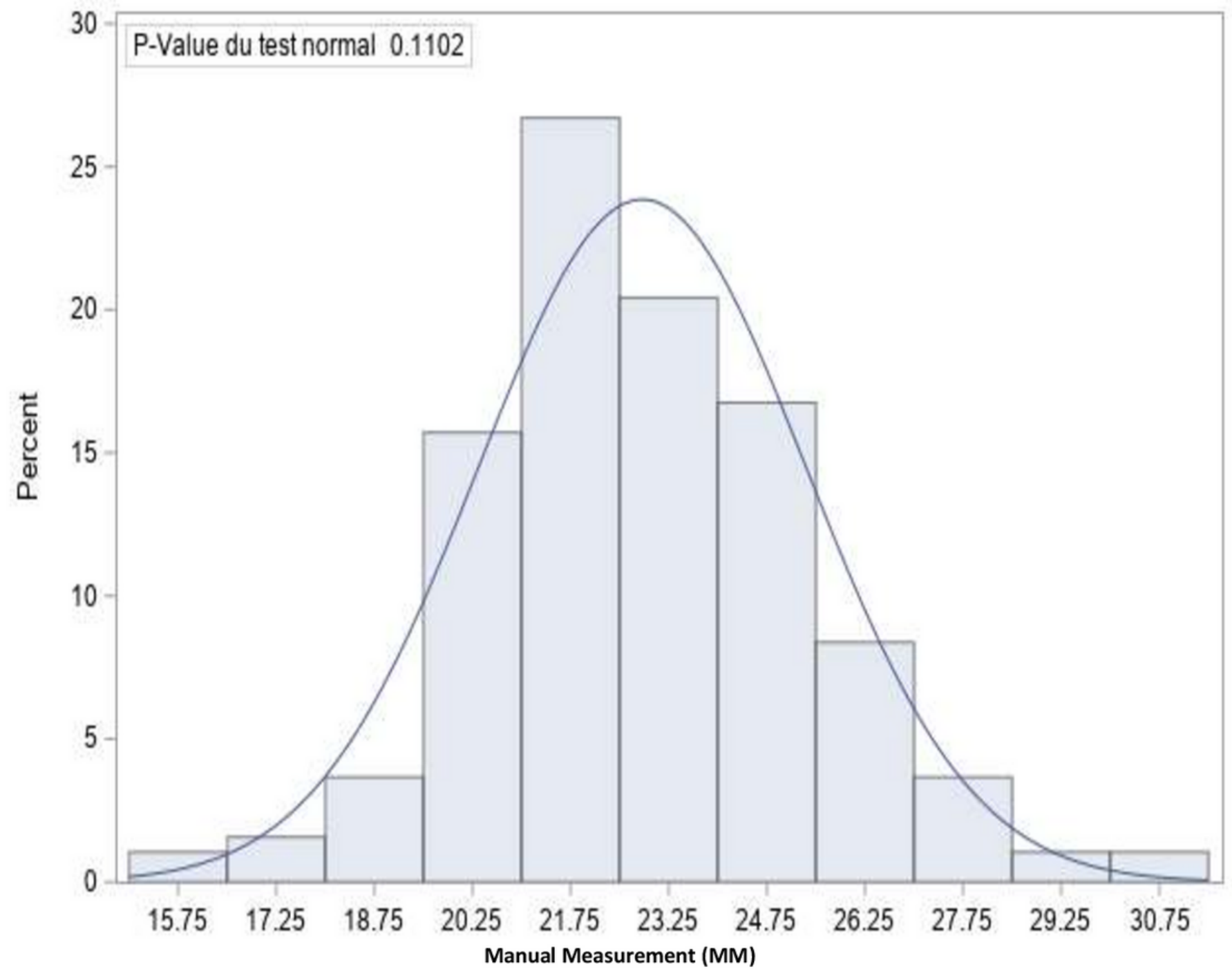

Figure 3

Distribution of Manual Measurements (millimeter) 


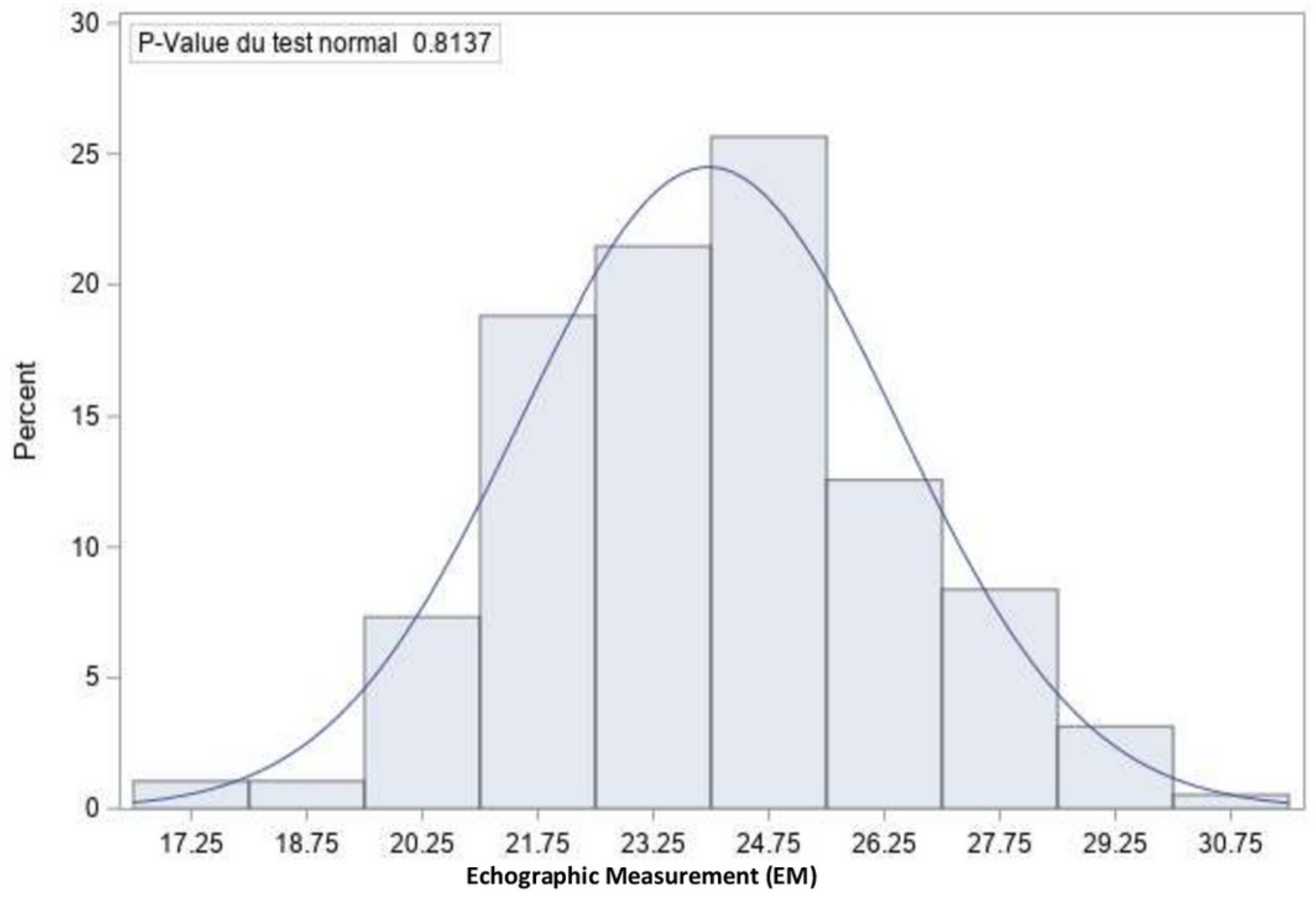

Figure 4

Distribution of Echographic Measurements (millimeter) 


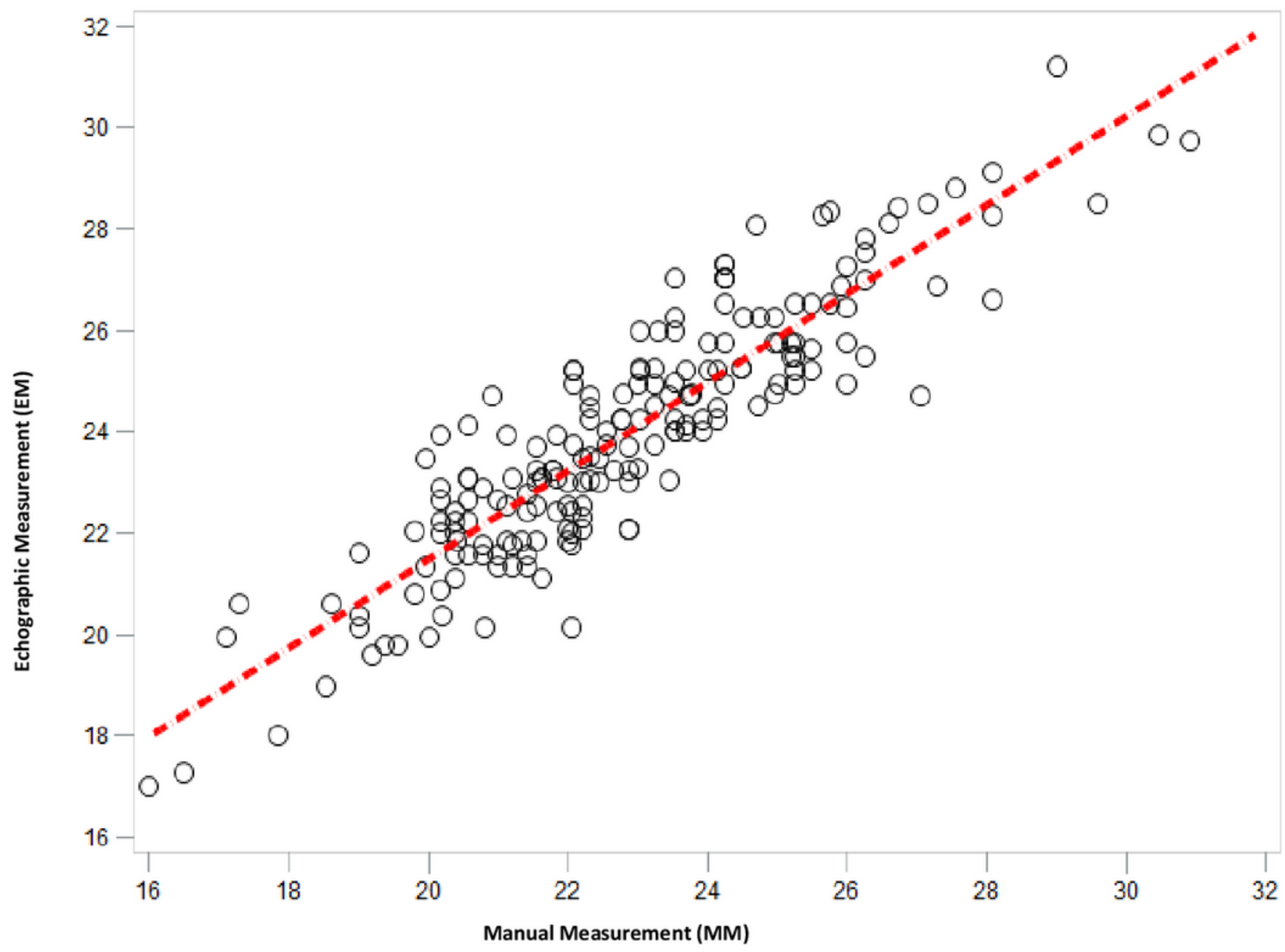

Figure 5

Correlation between Echographic and Manual Measurements (MM and EM) 

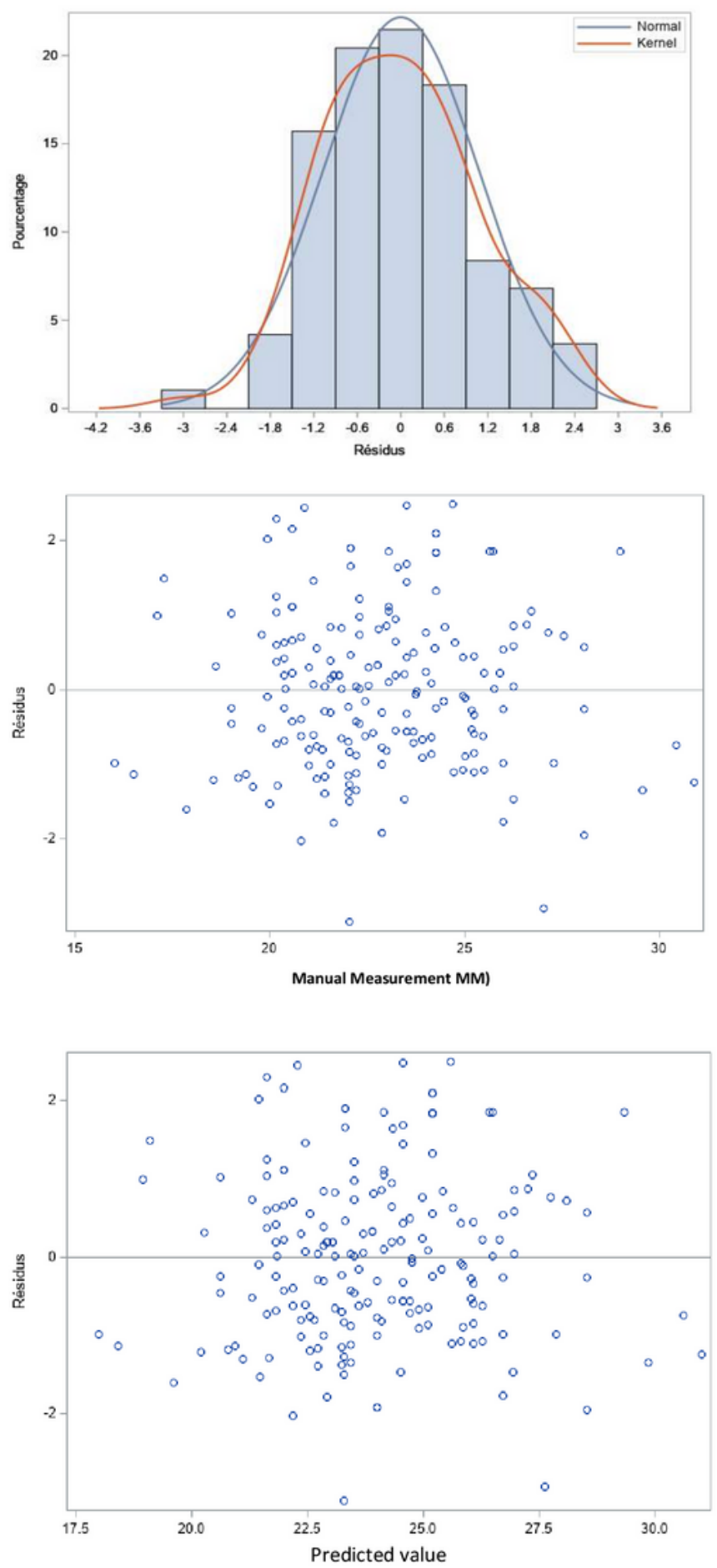

\section{Figure 6}

a Distribution of Echographic Measurement Residues (validate the model) b Residues of Echographic Measurements (validate the model) c Residues by predicted value for Echographic Measurement (validate the model) 


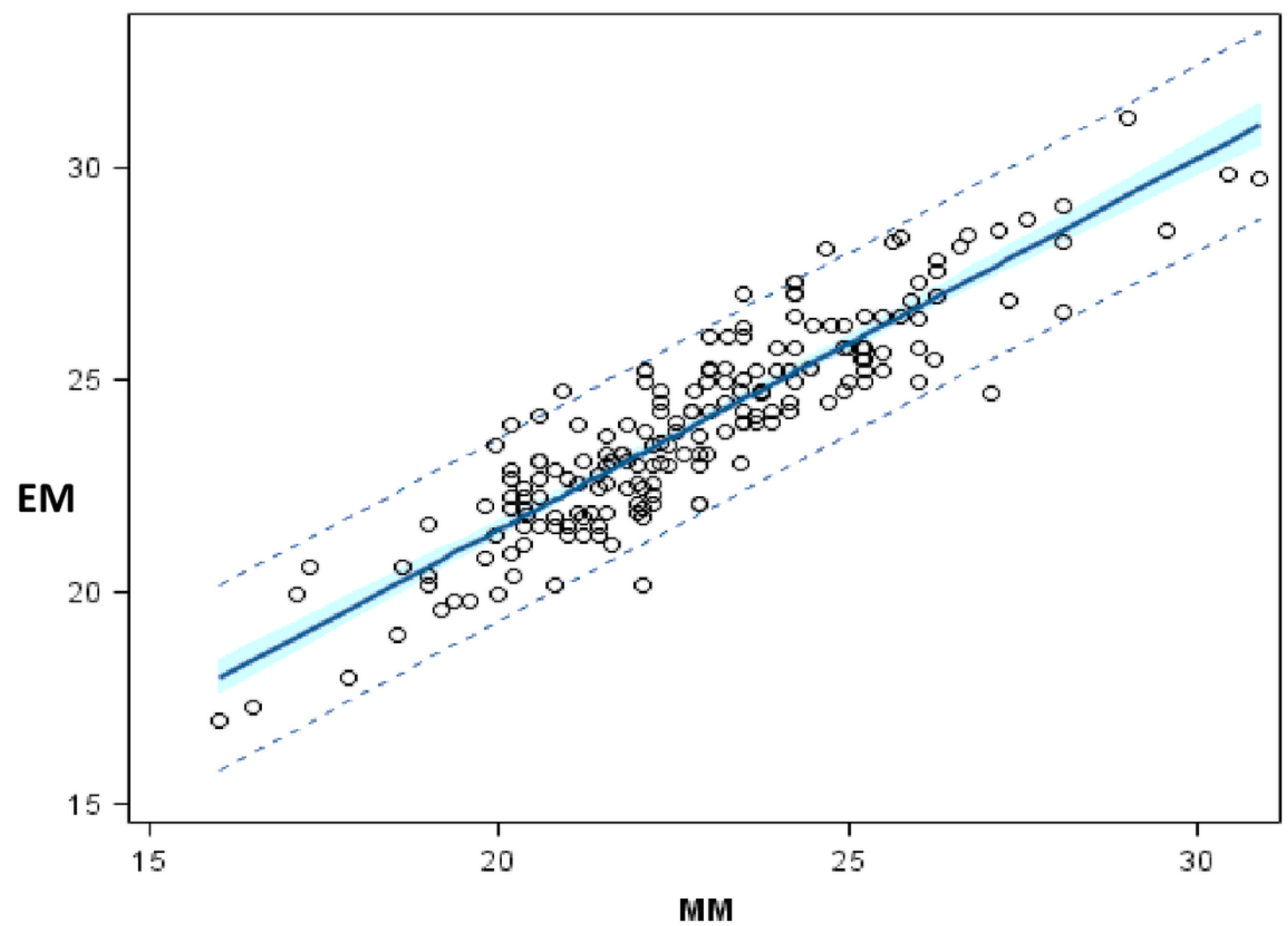

Figure 7

Adjustment curve for Echographic Measurements (ME) according to Manual Measurements (MM)

\section{Supplementary Files}

This is a list of supplementary files associated with this preprint. Click to download.

- TableCoefficientCorrelation.rtf 